\title{
A Genetic Algorithm Approach for the Identification of Microgrids Partitioning into Distribution Networks
}

\author{
Saman Korjani*, Angelo Facchini*†, Mario Mureddu*, Alfonso Damiano* \\ *Dipartimento di Ingegneria Elettrica ed Elettronica, \\ Università di Cagliari, Italy. \\ Via Marengo,1 09123 Cagliari \\ ${ }^{\dagger}$ IMT School for Advanced Studies Lucca, \\ Piazza S. Francesco 19 \\ 55100 Lucca, Italy \\ Email: saman.korjani@diee.unica.it
}

\begin{abstract}
In this paper a Genetic Algorithm (GA) is used to partition a distribution network with the aim to minimize the energy exchange among the microgrids (i.e. maximize selfconsumption) in presence of distributed generation. The proposed GA is tested on the IEEE prototypical network PG \& E 69-bus. The microgrid partitioning is tested over a period of one year with hourly sampled data of real household consumption and real distributed generation data. The proposed GA approach is compared with a Tabu Search (TS) method already presented in the scientific literature. Results show that both GA and TS lead to the identification of equivalent microgrids. However, the GA based approach achieves better convergence results allowing for a reliable network partitioning with less CPU effort. Moreover, the histograms of the power unbalances of the microgrids show unimodal and skewed distributions offering an interesting starting point for the appropriate deployment of storage and control systems.
\end{abstract}

\section{INTRODUCTION}

The use of high shares of Renewable Energy Sources (RES) for electricity production is widely considered as one of the key topics to decarbonize the electricity infrastructure, especially in cities (as also stated by the recent COP21 in Paris, 2015). Under the infrastructural point of view, the increasing adoption of RES poses a set of challenges to power systems, that need to become more flexible and resilient in order to manage intermittent energy sources like Photovoltaic (PV) and wind. In recent years, the deployment of electronic meters allowed for the progressive digitalization of the power infrastructures, enabling interoperability, integration, and automatic control of High and Medium Voltage (HV and MV) networks. Digital infrastructures also allow for the exploitation of the full potential of the microgrid (MG) concept, i.e. "a group of interconnected loads and distributed energy resources

This work is developed within the project NETfficient, "Energy and Economic Efficiency for Todays Smart Communities through Integrated Mult Storage Technologies". This project has received funding from the European Unions Horizon 2020 research and innovation programme under grant agreement No 646463. A.F. and M.M. gratefully acknowledge the Sardinia Regional Government for the financial support of this research activity developed under the European Regional Development Fund (ERDF) 2007-2013 P.O. Sardegna, within the following frameworks: Art. 15 Legge 241/90 "Attività di ricerca e sperimentazione nel settore delle smart grid e mobilità elettrica". within clearly defined electrical boundaries that acts as a single controllable entity with respect to the grid "[1]. Microgrids gained importance in the recent years because they allow for a better urban resilience and energy/emissions savings. Also, they constitute one of the emerging concepts for transforming the power networks in order to manage services like distributed generation and electric mobility [2].

In recent years different network partitioning methods have been proposed with the aim to identify the optimal partition of power grids in smaller microgrids. These include different optimization methods using DC power flow [3], complex networks [4]-[6], and intelligent systems [7]-[9].

Optimization problem can be also solved by means of bottom-up methods mimicking the laws of evolution: the Genetic Algorithms (GA). The concept of GA has been introduced by Holland [10] in the 80 s as a part of the computational techniques developed in the field of Artificial Life [11]. The foundational idea of GA is to generate high-quality solutions for optimization and search problems by applying to an initial population of solutions bio-inspired operators such as mutation, crossover and selection. The key concept is to initialize a population of randomly generated candidate solutions subject to the evolutionary pressure represented by a fitness function, i.e. a functional measuring the ability of each element of the population to solve the problem. The optimal solution is found by iterating the algorithm until the value of the fitness function converges to the equilibrium (usually after 30-100 iterations). GA have been successfully applied to different fields, ranging from bioinformatics, control engineering, neural networks, and medicine [12]. GA also found applications in power systems to find optimal Distributed Generation (DG) placement [13], reconfigure networks to minimize losses [14], and to reconfigure networks in case of failure [15].

In this paper a GA is developed for clustering a power network in which DG is present together with household/service users. The aim of the work is to find the optimal clusterization that minimizes the energy exchanges between the microgrids, or alternatively, the configuration maximizing the self consumption of the identified clusters. To this aim the GA is implemented to find microgrids partitioning in a prototypical 
IEEE network used as benchmark by [9], [16]. In order to validate the proposed approach the results have been compared with [9], [16]-[18], who implemented a Tabu Search (TS) method in presence of distributed generation. The analysis have been performed using a dataset composed by actual yearly profiles of load and RES, sampled hourly.

Results show that both GA and TS lead to similar outcomes, but with different convergence probability, being the GA-based algorithm better under this point of view, and allowing to consider the GA partitioning methods highly reliable. This paper is organized as follows: in section II are described data and methods used to implement the GA and the network used as benchmark, as well as the time series data used to compute the load in each node of the network. Section III describes the results, and in section IV conclusion and suggestions for future research are discussed.

\section{DESCRIPTION OF DATA AND METHODS}

The aim of the proposed methodology is to compute the best way to partition existing networks in microgrids. The goal is to minimize power exchange between them. Since the power time evolutions of loads and RES are intermittent, it is very hard to identify a network partitioning which ensures instantaneous balance among microgrids. For this reason, it is important to identify the optimal static configuration which minimizes the power exchanges between microgrids during a specific period of time. The starting point of the proposed methodology is an existing power grid, in which the position of both generation and load is known. Consequently, the topology of the network is used to identify the partitioning which minimize the power flow through the transmitting lines among the identified microgrids. In order to obtain this goal, the Fitness Function (FF) proposed in [9] has been used. This function is given in equation (1), where $N$ is number of the microgrids, and $P_{i}^{B}(t)$ and $Q_{i}^{B}(t)$ are the active and reactive power balance of microgrid $i$ at each time $t$, as defined in equations (2) and (3). The quantities $P_{i}^{L}(t), Q_{i}^{L}(t), P_{i}^{G}(t)$ and $Q_{i}^{G}(t)$ used in (2) and (3) are, respectively, the active and reactive consumption and production of each microgrid $i$ at time $t$.

$$
\begin{gathered}
F F=\frac{\sum_{t}^{T} \sum_{i}^{N}\left(P_{i}^{B}(t)\right)^{2}+\sum_{t}^{T} \sum_{i}^{N}\left(Q_{i}^{B}(t)\right)^{2}}{N \cdot T} \\
P_{i}^{B}(t)=P_{i}^{L}(t)-P_{i}^{G}(t) \\
Q_{i}^{B}(t)=Q_{i}^{L}(t)-Q_{i}^{G}(t)
\end{gathered}
$$

This implemented FF is the sum of mean squares of the active and reactive power exchanges among the microgrids. The minimization of this function is then made by means of a GA. Description of this methodology is given in subsec. II-A. In order to compare the results of the GA with [9], the optimization of the FF has been performed with the TS algorithm described in subsec. II-A.
The combination of microgrids minimizing the FF is then chosen as optimal one, and the distribution of active and reactive power balances during the year $\left\{P_{i}^{B}(t)\right\}$ and $\left\{Q_{i}^{B}(t)\right\}$ are obtained for each microgrid $i$. The result of optimal microgrid clustering and the obtained distributions of $\left\{P_{i}^{B}(t)\right\}$ and $\left\{Q_{i}^{B}(t)\right\}$ are given in sec. III.

\section{A. Optimization algorithms}

The flowchart of the GA is presented in Fig. 1. Initial

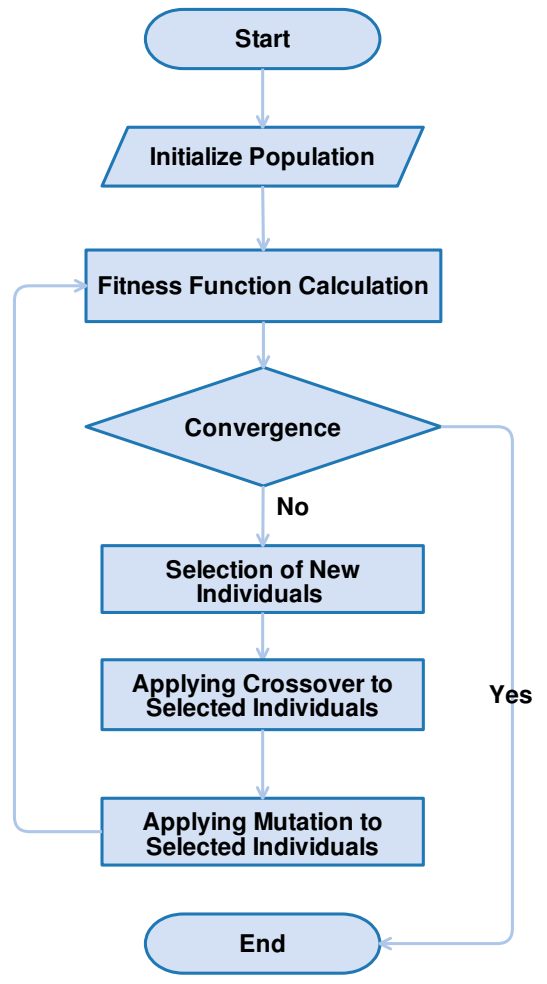

Fig. 1. The GA flowchart.

population is obtained by randomly creating a population of possible microgrid clusterings. The GA starts with population of $S$ possible configurations, considering a maximum of 6 microgrids. The maximum number of iterations is set to 100 . The crossover probability is set to $C=0.8$, while mutation probability is set to $M=0.2$.

In order to validate the GA optimization, the results have been compared with the TS used in the original work of Arefifar et al. [9]. TS is an AI-based heuristic optimization procedure introduced by Glover [19] implementing an iterative, random search of the solution space, visually described in Fig. 2. The name Tabu comes from the use of the so-called Tabu-List, in which the previously tested solutions are placed for a certain set of iterations $A_{t a b u}$. The algorithm is designed in a way that if it finds a previously evaluated solution during the random process, it skips it and considers another one unless it meets some conditions, called aspiration condition. In this case, the aspiration condition is achieved if the estimated solution gives a lower cost than the previous reference one, and the considered $A_{t a b u}$ has been set to 5 iterations. 


\section{B. Case study}

To test the proposed methodology on a benchmark case study, the standard PG \& E 69-bus radial distribution network is used [20]. This dataset has been chosen in order to compare the obtained results with the original paper of Arefifar et al. [9]. For this reason, the position of DGs and their rated power has been chosen accordingly to their paper. Since the power profiles used in [9] are not given, real yearly profiles for load and DG are used. The load data has been obtained by using the methodology described in [21], where the consumption profiles of Medium Voltage nodes is aggregated randomly, starting from a set of consumption time series of single households obtained from [21]. Following that procedure, it has been possible to create the yearly load profile of each node of the network, sampled hourly. The DG production considered in this study has been taken from the website of IESO, the Power System Operator based in Ontario (Canada) [22]. In this website, the hourly production data is given for each generator of the grid, from 2010 on. The dataset used in this paper covers the period from April 2016 to March 2017. From that, the production of wind and PV generators of comparable size with the ones used in this paper has been taken. Output power of biomass DGs is kept constant and equal to their rated power.

\section{RESULTS}

Fig. 4 and 5 show the clustering results for GA and TS, respectively. Results show that the node number 5 is located in different clusters in GA and TS. In particular, it has been moved from cluster no. 1 by GA to the no. 2 by TS. This is an unforeseen result, since node 5 has 0 production and

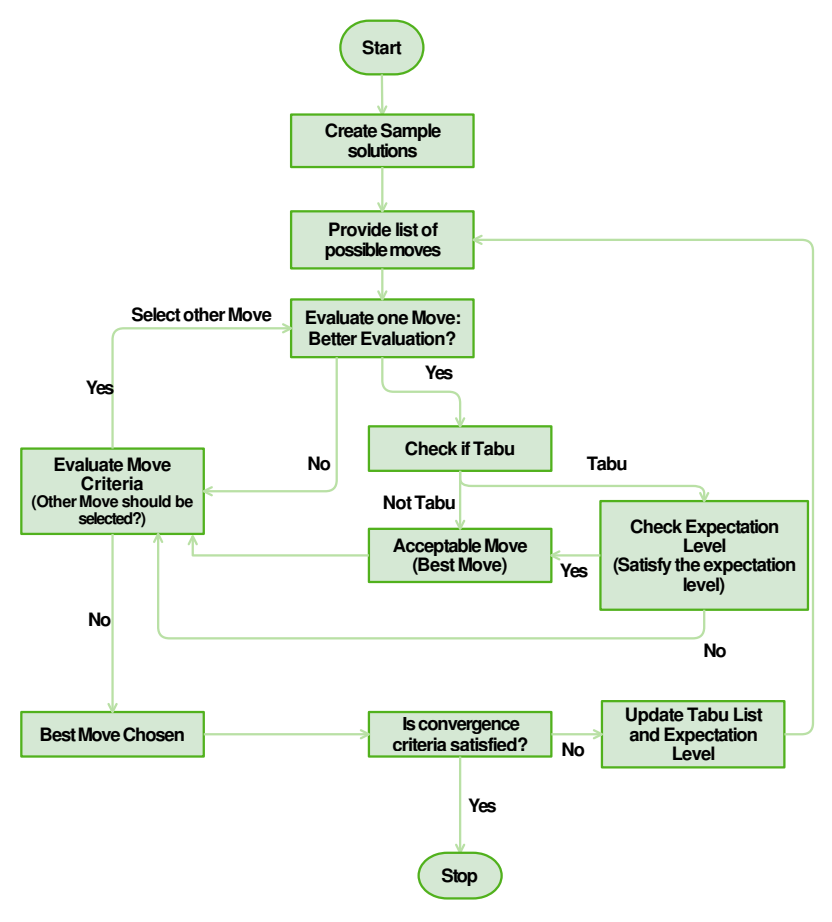

Fig. 2. The TS flowchart.

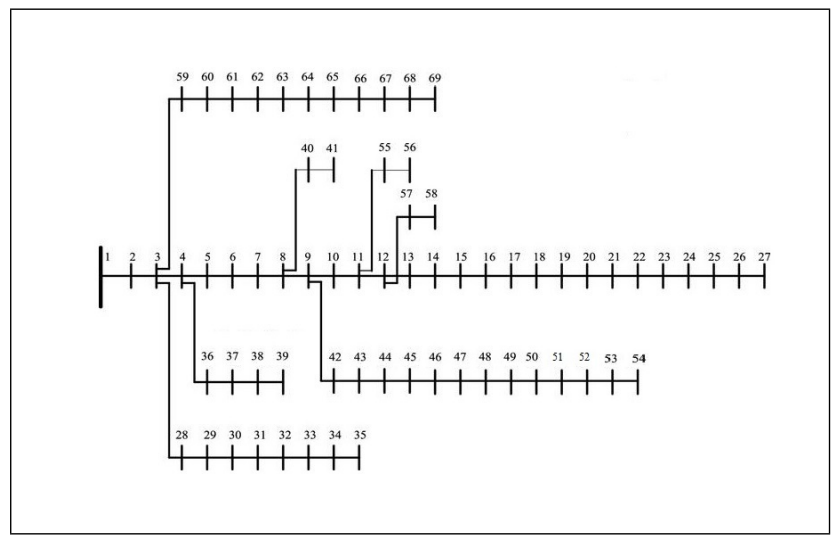

Fig. 3. The figure shows the IEEE 69 nodes network.

consumption, having no impact on the cost function. Because of this, every different clustering involving the change of node 5 position should be considered equivalent.

Fig. 6 shows the histograms representing the occurrence of different active power balances $P_{i}^{B}(t)$ in the obtained microgrids, during the entire year of the simulation. Negative results are associated to a net production, whereas positive are associated to net consumption. All the clusters show unimodal distributions, which can ease the implementation of control systems and techniques.

Given the problem-specific performances of heuristic optimization algorithms, a comparison between the convergence properties of TS and GA has been performed. The results are shown in fig. 7. The two algorithms have been run 100 times with values of sample size $S$ between 100 and 1000, in steps of 100 . The aim is to estimate the probability $p_{\text {best }}(S)$ for both algorithms to find the optimal solution. The relative error associated to each probability has been calculated as the square root of the number of trials. In general, the best cost function calculated by TS and GA has exactly the same value, but the $p_{\text {best }}(S)$ values differ for the two algorithms. In particular, Fig. 7 clearly shows how the GA ensures in general the best convergence properties reaching the $100 \%$ value starting from a population size of 400 , leading to more stable results. In this case, the better performance of the GA may be due to a better spanning of the solution space with respect to the TS implementation.

\section{Discussion And Conclusions}

In this paper Genetic Algorithms are used for the identification of the optimal positioning of microgrids in an existing power grid with DG. The aim is to obtain a partition minimizing the power exchange between the microgrids in time (i.e. maximizing self consumption). The GA has been tested on the PG\&E 69 bus network, in which the load and generation profiles are derived by real consumption and production time series hourly sampled and measured for a period of one year. As a first result, the GA algorithm shows the ability to identify the optimal partitioning of the network. The results are consistent with those obtained by the TS algorithm already 


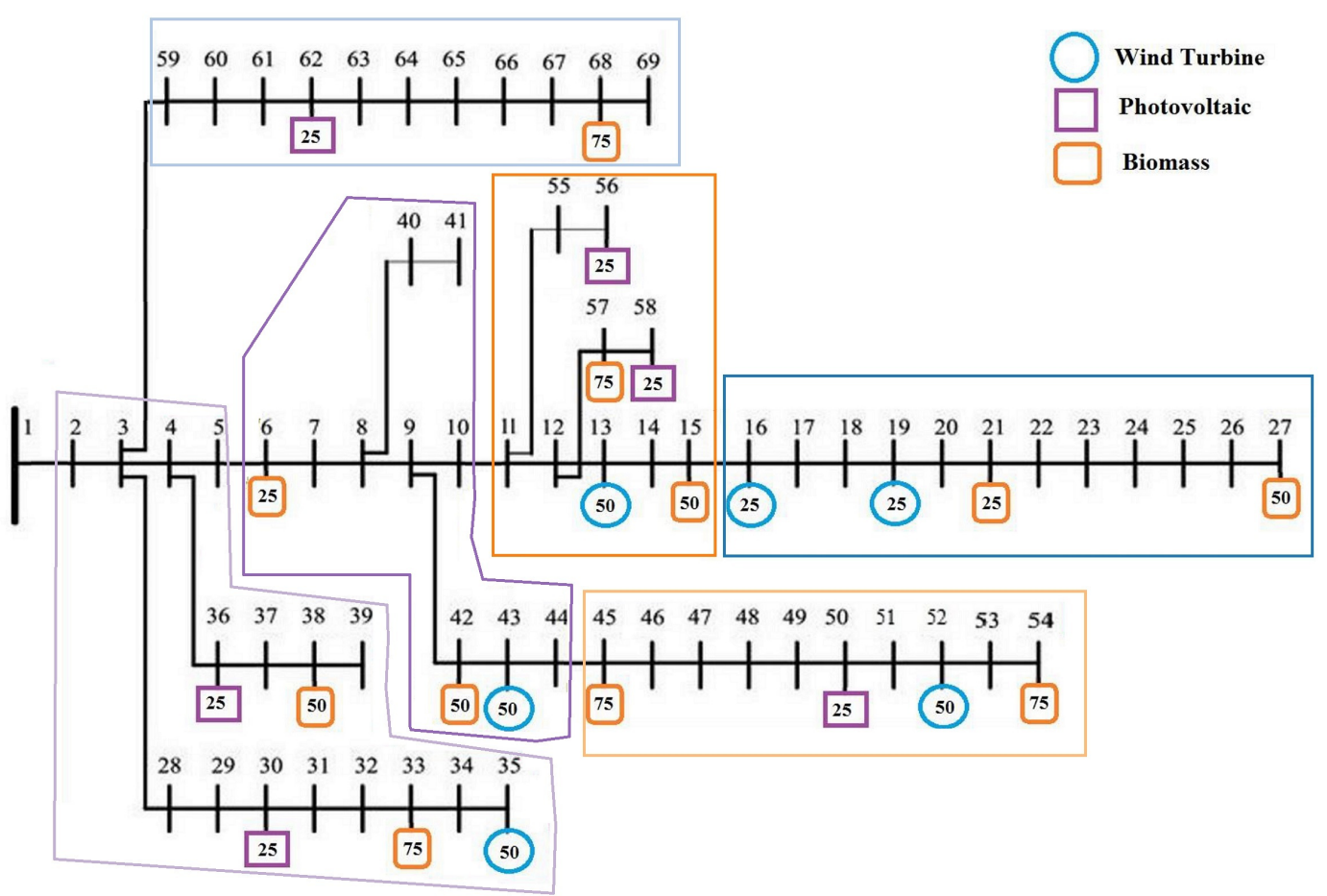

Fig. 4. Optimal clustering obtained with the GA algorithm.

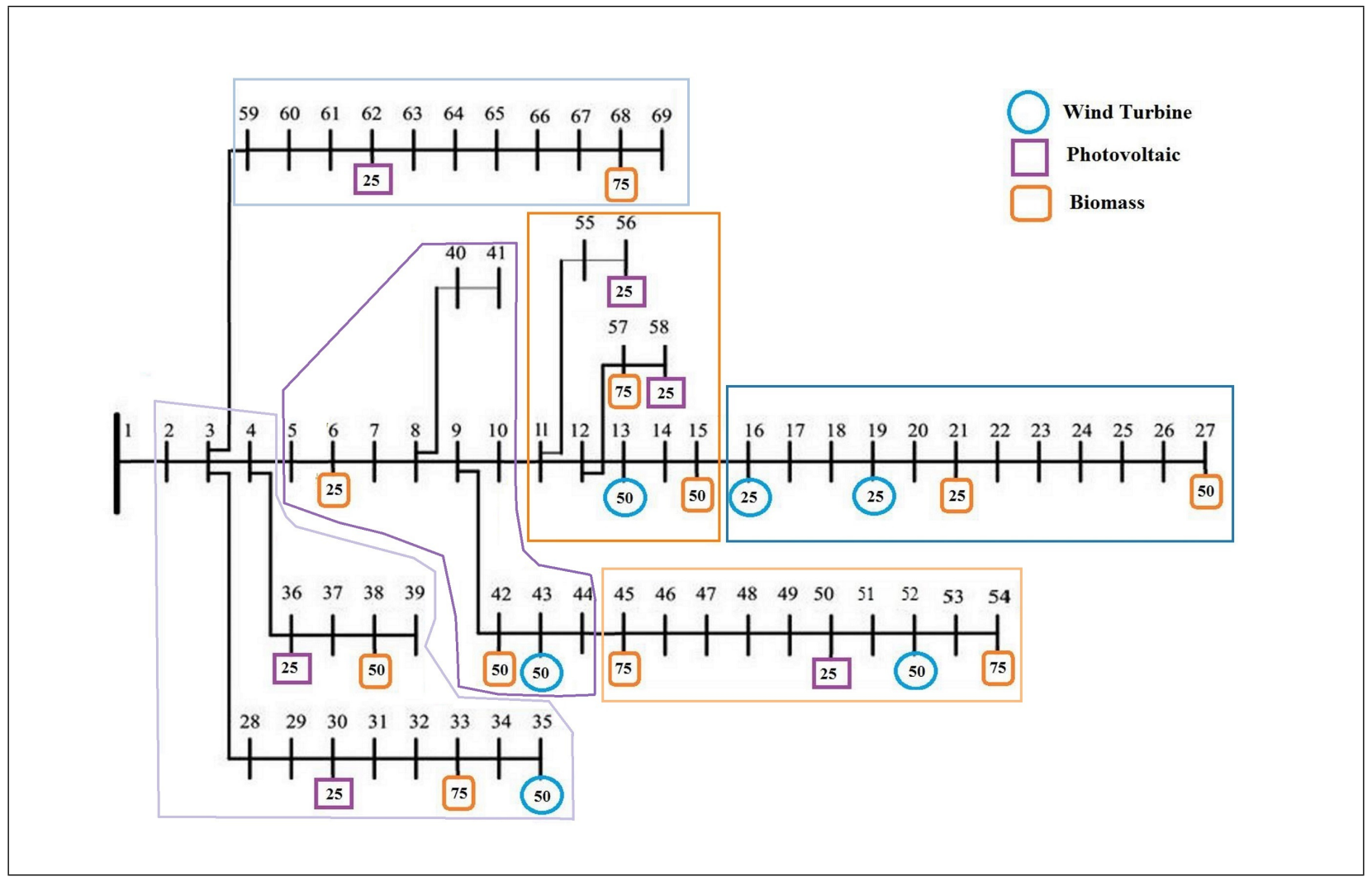

Fig. 5. Optimal clustering obtained with the TS algorithm. 


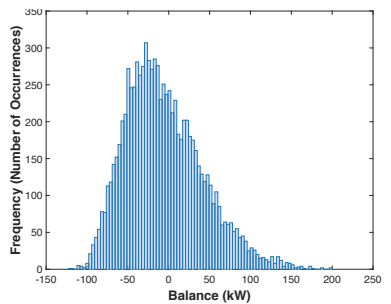

(a) Micro grid 1

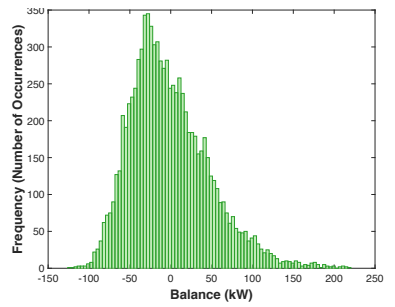

(c) Micro grid 3

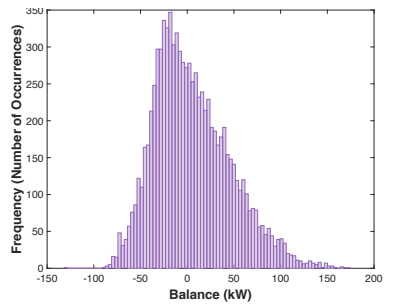

(e) Micro grid 5

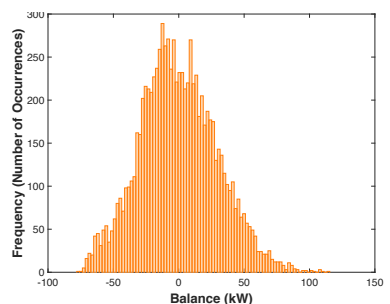

(b) Micro grid 2

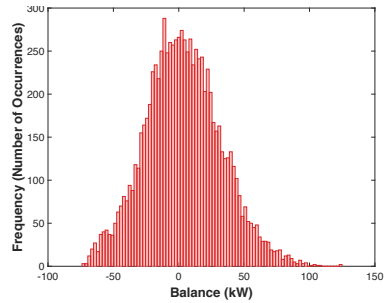

(d) Micro grid 4

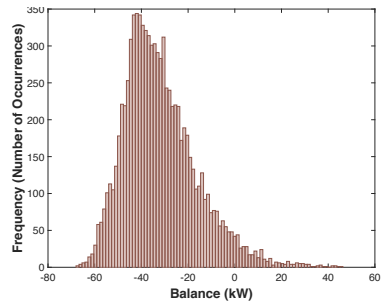

(f) Micro grid 6
Fig. 6. Histograms showing the distribution of active balances $P^{B}(t)$ of the obtained microgrids.

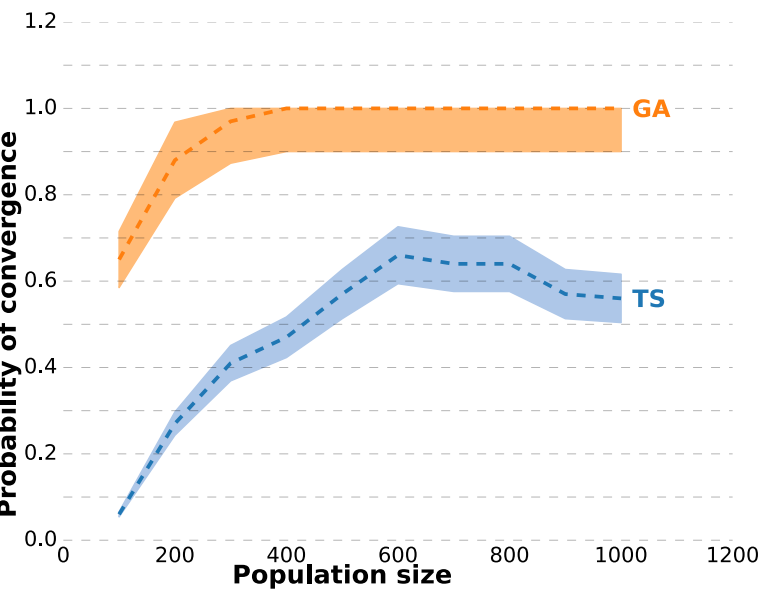

Fig. 7. Probability of convergence vs population size for Tabu Search (blue) and Genetic Algorithm (orange).

used in literature. However, the GA shows better convergence properties with respect to TS. The proposed approach leads to more stable solutions and to less CPU effort.

Furthermore, the shapes of the distribution of the power unbalances of each microgrid have been computed. Results show unimodal, skewed distributions, suggesting an interesting starting point for the appropriate deployment of storage and control systems.

Future works will be devoted to include storage systems in the network and to a better characterization of the power unbalances fluctuations.

\section{REFERENCES}

[1] R. Lasseter and P. Paigi, "Microgrid: a conceptual solution," in 2004 IEEE 35th Annual Power Electronics Specialists Conference (IEEE Cat. No.04CH37551). IEEE, pp. 4285-4290.

[2] N. Hatziargyriou, Microgrids Architectures and Control. John Wiley and Sons Ltd., 2014.

[3] T. Morstyn, B. Hredzak, and V. G. Agelidis, "Dynamic Optimal Power Flow for DC Microgrids with Distributed Battery Energy Storage Systems," 2016 IEEE Energy Conversion Congress and Exposition (ECCE), vol. 3053, no. c, pp. 1-6, 2016.

[4] A. Halu, A. Scala, A. Khiyami, and M. C. González, "Data-driven modeling of solar-powered urban microgrids." Science advances, vol. 2, no. 1, p. e1500700, 2016

[5] L. Valori, G. L. Giannuzzi, A. Facchini, T. Squartini, D. Garlaschelli, and R. Basosi, "A generation-attraction model for renewable energy flows in Italy: A complex network approach," European Physical Journal: Special Topics, vol. 225, no. 10, pp. 1913-1927, oct 2016.

[6] M. Mureddu, G. Caldarelli, A. Damiano, A. Scala, and H. MeyerOrtmanns, "Islanding the power grid on the transmission level: less connections for more security," Scientific Reports, vol. 6, p. 34797, oct 2016.

[7] M. Simonov, "Dynamic partitioning of DC microgrid in resilient clusters using event-driven approach," IEEE Transactions on Smart Grid, vol. 5, no. 5, pp. 2618-2625, 2014.

[8] M. A. Alotaibi and M. M. A. Salama, "An efficient probabilisticchronological matching modeling for DG planning and reliability assessment in power distribution systems," Renewable Energy, vol. 99, no. October, pp. 158-169, 2016.

[9] S. A. Arefifar, Y. A. R. I. Mohamed, and T. H. M. El-Fouly, "Optimum microgrid design for enhancing reliability and supply-security," IEEE Transactions on Smart Grid, vol. 4, no. 3, pp. 1567-1575, 2013.

[10] J. H. Holland, Adaptation in Natural and Artificial Systems. MIT Press, 1992.

[11] C. G. L. (ed.), Atificial Life: An overview. MIT Press, 1997.

[12] L. Chambers, The Practical Handbook of Genetic Algorithms: Applications, Second Edition. Chapman\&Hall/CRC, 2000.

[13] P. Georgilakis and N. Hatziargyriou, "Optimal distributed generation placement in power distribution networks: models, methods, and future research," IEEE Transactions on Power Systems, vol. 28, no. 3, pp. 3420-3428, 2013.

[14] J. C. Cebrian and N. Kagan, "Reconfiguration of distribution networks to minimize loss and disruption costs using genetic algorithms," Electric Power Systems Research, vol. 80, no. 1, pp. 53-62, 2010.

[15] T. Lu, Z. Wang, Q. Ai, and W.-J. Lee, "Interactive Model for Energy Management of Clustered Microgrids," IEEE Transactions on Industry Applications, vol. 9994, no. c, pp. 1-1, 2017.

[16] S. A. Arefifar, Y. A. R. I. Mohamed, and T. H. M. El-Fouly, "Supplyadequacy-based optimal construction of microgrids in smart distribution systems," IEEE Transactions on Smart Grid, vol. 3, no. 3, pp. 14911502, 2012.

[17] S. A. Arefifar and Y. A.-r. I. Mohamed, "DG Mix , Reactive Sources and Energy Storage Units for Optimizing Microgrid Reliability and Supply Security," IEEE Transactions on Smart Grid, vol. 5, no. 4, pp. 1-10, 2014.

[18] S. A. Arefifar, Y. A.-r. I. Mohamed, and T. El-fouly, "Optimized Multiple Microgrid-Based Clustering of Active Distribution Systems Considering Communication and Control Requirements," IEEE Transactions on Industrial Electronics, vol. 62, no. 2, pp. 711-723, 2015.

[19] F. Glover, "Artificial intelligence, heuristic frameworks and tabu search," Managerial and Decision Economics, vol. 11, no. 5, pp. 365-375, 1990.

[20] M. E. Baran and F. F. Wu, "Optimal capacitor placement on radial distribution systems," IEEE Transactions on Power Delivery, vol. 4, no. 1, pp. 725-734, 1989.

[21] A. R. Malekpour and A. Pahwa, "Radial Test Feeder including primary and secondary distribution network," 2015 North American Power Symposium (NAPS), pp. 1-9, 2015.

[22] IESO, "IESO," 2017. [Online]. Available: http://ieso.ca/ 